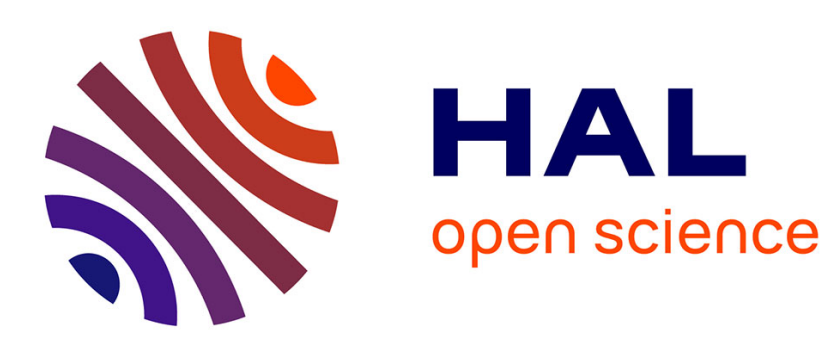

\title{
Synthesis and characterization of new pyridine-based chiral calamitic liquid crystals
}

Deniz Vardar, Hale Ocak, Huriye Akdas Kilic, Olivier Jeannin, Franck Camerel, Belkiz Bilgin Eran

\section{- To cite this version:}

Deniz Vardar, Hale Ocak, Huriye Akdas Kilic, Olivier Jeannin, Franck Camerel, et al.. Synthesis and characterization of new pyridine-based chiral calamitic liquid crystals. Liquid Crystals, 2021, 48 (6), pp.850-861. 10.1080/02678292.2020.1825841 . hal-02996418

\section{HAL Id: hal-02996418 \\ https://hal.science/hal-02996418}

Submitted on 13 Oct 2021

HAL is a multi-disciplinary open access archive for the deposit and dissemination of scientific research documents, whether they are published or not. The documents may come from teaching and research institutions in France or abroad, or from public or private research centers.
L'archive ouverte pluridisciplinaire HAL, est destinée au dépôt et à la diffusion de documents scientifiques de niveau recherche, publiés ou non, émanant des établissements d'enseignement et de recherche français ou étrangers, des laboratoires publics ou privés. 


\title{
Synthesis and Characterization of New Pyridine Based Chiral Calamitic Liquid Crystals
}

Deniz $\operatorname{Vardar}^{1}$, Hale Ocak ${ }^{1 *}$, Huriye Akdaş-Kılıç ${ }^{1,2}$, Olivier Jeannin ${ }^{2}$, Franck Camerel$^{2}$, Belkız Bilgin $\operatorname{Eran}^{1 *}$

${ }^{1}$ Yildiz Technical University, Department of Chemistry, 34220 Esenler, Istanbul, Turkey

${ }^{2}$ Univ Rennes, CNRS, ISCR - UMR 6226, F-35000 Rennes

*Corresponding Author: bbilgin@yildiz.edu.tr, hocak@yildiz.edu.tr

\begin{abstract}
:
In this paper, novel chiral calamitic compounds, consisting of $n$-dodecyloxyphenyl substituted pyridine carboxylate core linked to one or two benzene rings through ester linkers have been synthesized by multi-step procedures. The other end has been varied by introducing a flexible (S)-2-methylbutyloxy or $(S)$-3,7-dimethyloctyloxy chiral chain. The liquid crystal properties of new compounds were investigated by various experimental techniques such as DSC, POM and SAXS. The pyridine-based calamitic compounds exhibited enantiotropic chiral smectic mesophases such as $\mathrm{SmC}^{*}, \mathrm{SmA}$ or uncharacteristic mesophase depending on hetero aromatic rings such as nicotinate or picolinate cores, number of aromatic rings and the type of chiral branched chain.
\end{abstract}

Keywords: Liquid crystal, pyridine, calamitic compounds, nicotinate, picolinate, chiral chain. 


\section{Introduction}

A huge interest in the field of liquid crystals has been grown rapidly due to their unique properties which have led to useful and technologically promising materials. ${ }^{1,2,3,4}$ This special class of materials, which keep a combination of order and mobility at the molecular level, are used in many fields such as electro-optical displays and temperature sensors., ${ }^{5,6}$

The use of six-membered N-heterocyclic systems in mesogenic molecules impacts the mesomorphic properties due to the fact that the introduction of nitrogen atom in the molecular structures mainly effect the molecular geometry, polarity and polarisability. ${ }^{7}$ Pyridine is one of the simplest heterocyclic aromatics used as a core system in the design of liquid crystalline molecules. ${ }^{7,8,9,10,11}$ The position of the nitrogen atom in the molecular core considerably influences the liquid crystalline properties of pyridine derivatives ${ }^{12}$ and has a significant impact on the dispersion forces, the dipole moment, the polarizability of the molecule and inevitably, on the dielectric nature of the mesogen. ${ }^{13}$ Additionally, the geometry and the value of the dihedral angle between the two aromatic rings of the phenyl-pyridine moiety, ${ }^{14}$ depending of the position of the nitrogen, give rise to an effect on the mesophase molecular stacking. For this purpose, interesting mesomorphic behaviors are revealed by using pyridine ring in the central unit in calamitic molecules.

Recently, a variety of pyridine containing liquid crystalline compounds were synthesized, ${ }^{7,15,16,17}$ most of them were rod-like calamitic liquid crystals, but only few examples presenting nicotinate and picolinate as building blocks were reported, in order to determine the effect of 2-, 3-, and 4-pyridyl rings relative to phenyl in promoting mesophase thermal stability. ${ }^{10,18}$ However, several Japanese patents were published concerning optically active liquid crystal based on pyridine carboxylate, demonstrating the importance of this type of patterns for technological applications. ${ }^{19,20,21}$ The mentioned compounds in that patents mainly exhibit two liquid crystal mesophases such as SmC and SmA. They especially promise a potential to be used in display devices.

Previously, various biphenyl esters with a rod-like core, with interesting properties, were prepared and studied by optical and thermal methods. ${ }^{22,23}$ Recently, we reported the compounds that the internal phenyl ring of the biphenyl system replaced with a 3-pyridyl ring to generate functional materials with an interesting mesogenic behavior. ${ }^{24}$ Herein, we report the synthesis and the mesomorphic properties of novel chiral calamitic mesogens, consisting of $n$-dodecyloxyphenyl substituted pyridine carboxylate core linked to one or two benzene rings through ester linkers. Into the other terminal, a flexible $(S)$-2-methylbutyloxy or $(S)-3,7$ - 
dimethyloctyloxy chiral chain was introduced to focus on the effect of molecular chirality on mesophases of pyridine-based calamitic molecules.

\section{Experimental}

\subsection{Materials and instrumentation}

New pyridine-based chiral calamitic molecules $(\mathbf{4}, \mathbf{5}, \mathbf{7 a}, \mathbf{b}, \mathbf{1 0 a}, \mathbf{b}$ and 11a,b) were characterized by ${ }^{1} \mathrm{H}$ NMR and ${ }^{13} \mathrm{C}$ NMR (Bruker Avance III 500 spectrometer, in $\mathrm{CDCl}_{3}$ solution, with tetramethylsilane as internal standard). Microanalysis was performed using a Thermo Fischer Scientific FlashEA 1112 Series elemental analyzer. Fourier Transform Infrared (FT-IR) spectra of the newly synthesized molecules were recorded on a "Perkin Elmer Spectrum One".

The mesomorphic properties of compounds were investigated by using a Mettler FP-82 HT hot stage with its control unit coupled with a Leica DM2700P polarizing microscope. DSCthermograms of chiral pyridine derivatives were recorded on a Perkin-Elmer DSC-6, heating and cooling rate: $10{ }^{\circ} \mathrm{C} \min ^{-1}$ in a nitrogen atmosphere.

X-ray scattering experiments (SAXS) were performed using a Microstar Bruker rotating anode X-ray generator operated at $40 \mathrm{kV}$ and $40 \mathrm{~mA}$ with monochromatic $\mathrm{Cu} \mathrm{K} \alpha$ radiation ( $\lambda$ $=1.541 \AA$ ) and point collimation. The patterns were collected with a Mar345 Image-Plate detector (Marresearch, Norderstedt, Germany). The exposure time at each temperature was $3600 \mathrm{~s}$ and the heating or cooling speed between two temperatures was $10{ }^{\circ} \mathrm{C} / \mathrm{min}$. The samples were held in Lindeman glass capillaries $(1.5 \mathrm{~mm}$ diameter). The capillaries were placed inside a Linkam HFX350-Capillary X-Ray stage which allow measurements from -196 ${ }^{\circ} \mathrm{C}$ up to $350{ }^{\circ} \mathrm{C}$ with an accuracy of $0.1^{\circ} \mathrm{C}$.

Methyl 5-bromopyridine-2-carboxylate (ABCR), methyl 6-chloropyridine-3-carboxylate (Aldrich), 1-bromododecane (Merck), 4-bromophenol (Alfa Aesar), $\mathrm{K}_{2} \mathrm{CO}_{3}$ (Merck), nButyllithium solution $2.5 \mathrm{M}$ in hexanes (Alfa Aesar), trimethyl borate $\geq 98 \%$ (Alfa Aesar), hydrochloric acid technical grade, tetrakis(triphenylphosphine)-palladium (0) (Aldrich), 1,2Dimethoxyethane anhydrous $99.5 \%$ (Merck), sodium hydrogen carbonate (Merck), were purchased from commercial sources. 2-Butanone (Merck) and THF for HPLC, $\geq 99.9 \%$ (Riedel de Häen) were purchased commercially as dry solvents and were used without further purification. Solvents used in the purification step such as crystallization and column chromatography (hexane, ethyl acetate, chloroform, dichloromethane and ethanol) were distilled prior to use. Analytical thin-layer chromatography (TLC) was carried out on 
aluminium plates coated with silica gel 60 F254 (Merck). Column chromatography was performed using silica gel 60 (Merck, pore size $60 \AA$, 230-400 mesh particle size).

\subsection{Synthesis and Characterization}

The synthesis of new pyridine based chiral calamitic compounds $(\mathbf{4}, \mathbf{5}, \mathbf{7 a}, \mathbf{b}, \mathbf{1 0 a}, \mathbf{b}$ and $\mathbf{1 1 a}, \mathbf{b})$ are presented in Scheme 1. The main feature of these compounds is the presence of a picolinate or a nicotinate aromatic ring within the core.

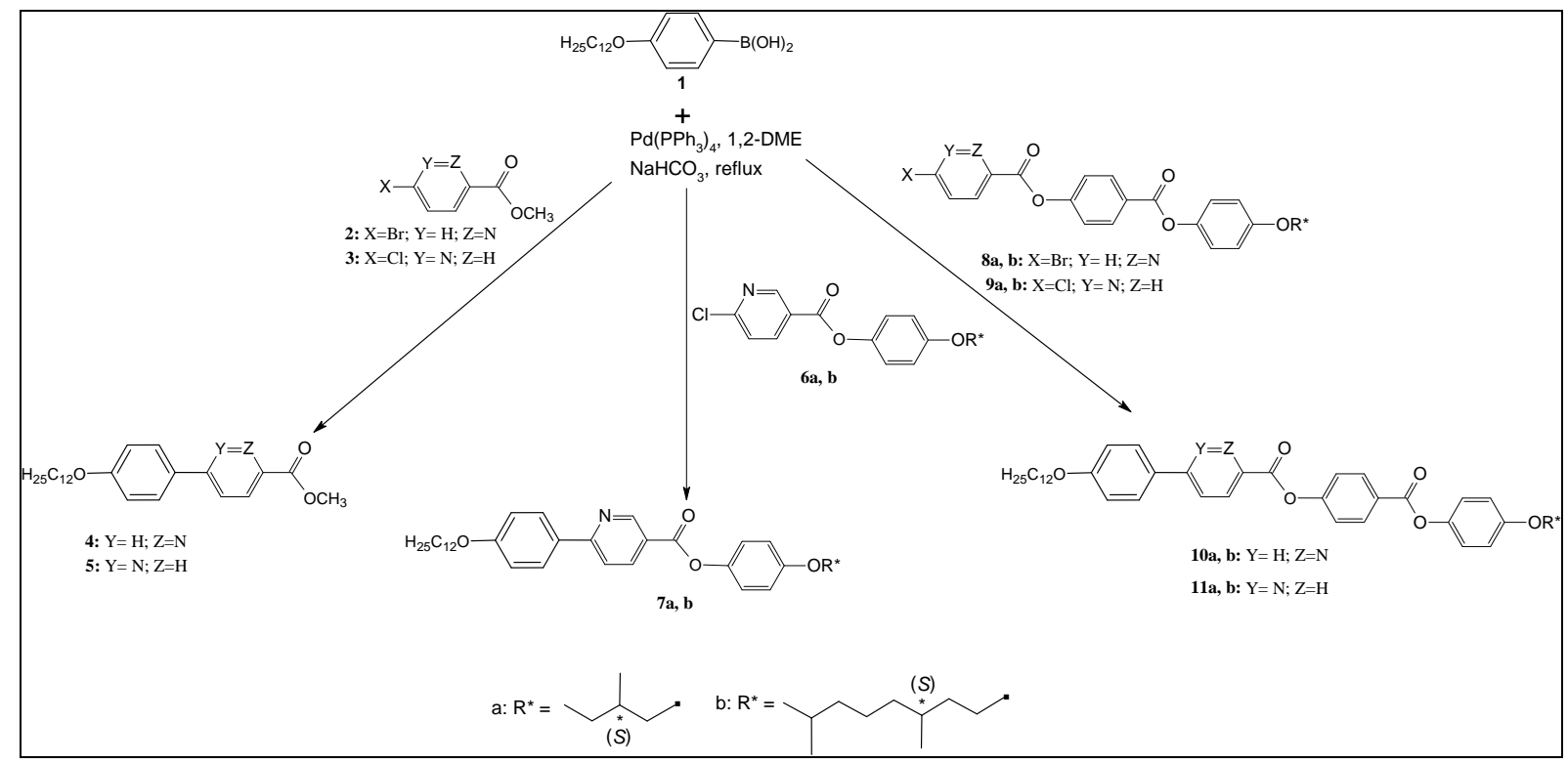

Scheme 1. Synthesis of the pyridine-based chiral calamitic compounds $\left(4^{24}, \mathbf{5}, \mathbf{7 a}, \mathbf{b}, \mathbf{1 0 a}, \mathbf{b}\right.$ and 11a,b).

The synthesis of the precursor molecules $\mathbf{6 a}, \mathbf{b}, \mathbf{8 a}, \mathbf{b}$ and $\mathbf{9 a , b}$ via using multi-step procedures, their characterizations and the mesomorphic properties were reported in our previous study. ${ }^{25}$ 4-Dodecyloxybenzen boronic $\operatorname{acid}^{26}$ (1) was synthesized starting from 1-bromo-4dodecyloxybenzen ${ }^{26}$ by using previously reported procedure. ${ }^{24,26}$ Methyl 5-bromopyridine-2carboxylate (2) and methyl 6-chloropyridine-3-carboxylate (3) were commercially purchased. Suzuki Cross-coupling reaction between the initial halogenated derivatives $(\mathbf{2}, \mathbf{3}, \mathbf{6 a}, \mathbf{b}, \mathbf{8 a}, \mathbf{b}$ and 9a,b) and the boronic acid $\mathbf{1}$ gave the target pyridine-based chiral calamitic compounds $\mathbf{4}$, $\mathbf{5}, \mathbf{7 a}, \mathbf{b}, \mathbf{1 0 a}, \mathbf{b}$ and 11a,b by using tetrakis(triphenylphosphine)-palladium (0) as catalyst in 1,2-dimethoxyethane. Spectroscopic data and mesomorphic properties of methyl 5-(4- $n$ dodecyloxyphenyl)pyridine-2-carboxylate (4) are given in ref. ${ }^{27}$. 
The general procedure for the synthesis of the target compounds $4,5,7 a, b, 10 a, b$ and 11a,b:

$0.5 \mathrm{mmol}$ 4-Dodecyloxybenzene boronic acid (1), $0.5 \mathrm{mmol}$ the initial halogenated derivatives $(\mathbf{2}, \mathbf{3}, \mathbf{6 a}, \mathbf{b}, \mathbf{8 a}, \mathbf{b}$ and $\mathbf{9 a}, \mathbf{b})$ and $0.022 \mathrm{mmol} \mathrm{Pd}\left(\mathrm{PPh}_{3}\right)_{4}$ were dissolved in $10 \mathrm{~mL}$ of 1,2-dimethoxyethane under argon atmosphere. After the addition of $0.75 \mathrm{~mL}$ of saturated $\mathrm{NaHCO}_{3}$ solution, the reaction mixture was boiled under reflux for 2.5 hours and the evolution was monitored by TLC (H: EA/3:1). The solvent was removed under reduced pressure and the residual mixture was extracted three times with $\mathrm{CHCl}_{3}$. The combined organic phases were washed with saturated $\mathrm{NaCl}$ solution and dried over $\mathrm{Na}_{2} \mathrm{SO}_{4}$. The solvent was evaporated in a rotary evaporator. The residue was dissolved in $\mathrm{CHCl}_{3}$ and filtered over silica gel in order to remove the catalyst from the medium. The product was purified by column chromatography (silica gel 60, H: EA/3:1).

\section{Methyl 6-(4-dodecyloxyphenyl)pyridine-3-carboxylate (5)}

Yield: $42 \%$; colorless crystals. ${ }^{1} \mathbf{H}$ NMR $\left(500 \mathrm{MHz} \mathrm{CDCl}_{3}\right): \delta(\mathrm{ppm})=9.24(\mathrm{~d}, J \approx 2.1 \mathrm{~Hz}$, $1 \mathrm{H}, \mathrm{Ar}-\mathrm{CH}), 8.31\left(\mathrm{dd}, J_{l} \approx 8.4 \mathrm{~Hz}, J_{2} \approx 2.2 \mathrm{~Hz} 1 \mathrm{H}, \mathrm{Ar}-\mathrm{CH}\right), 8.03\left(\mathrm{~d}, J_{l} \approx 8.9 \mathrm{~Hz}, 2 \mathrm{H}, \mathrm{Ar}-\mathrm{CH}\right.$ ), $7.76(\mathrm{~d}, J \approx 8.4 \mathrm{~Hz}, 1 \mathrm{H}, \mathrm{Ar}-\mathrm{CH}), 7.02(\mathrm{~d}, J \approx 8.9 \mathrm{~Hz}, 2 \mathrm{H}, \mathrm{Ar}-\mathrm{CH}), 4.04(\mathrm{t}, J \approx 6.5 \mathrm{~Hz}, 2 \mathrm{H}$, $\left.\mathrm{OCH}_{2}\right), 3.98\left(\mathrm{~s}, 3 \mathrm{H}, \mathrm{OCH}_{3}\right), 1.86-1.77\left(\mathrm{~m}, 2 \mathrm{H}, \mathrm{CH}_{2}\right), 1.61-1.25\left(\mathrm{~m}, 18 \mathrm{H}, \mathrm{CH}_{2}\right), 0.89(\mathrm{t}, J \approx$ $\left.6.5 \mathrm{~Hz}, 3 \mathrm{H}, \mathrm{CH}_{3}\right) .{ }^{13} \mathrm{C}$ NMR $\left(125 \mathrm{MHz}, \mathrm{CDCl}_{3}\right): \delta(\mathrm{ppm})=166.00(\mathrm{COO}), 160.95,160.56$, 130.44, 123.36 (Ar-C), 150.86, 137.80, 128.76, 118.91, $114.83(\mathrm{Ar}-\mathrm{CH}), 68.18\left(\mathrm{OCH}_{2}\right), 52.26$ $\left(\mathrm{OCH}_{3}\right), 31.92,29.66,29.64,29.60,29.58,29.40,29.35,29.21,26.02,22.69\left(\mathrm{CH}_{2}\right), 14.12$ $\left(\mathrm{CH}_{3}\right)$. FT-IR: $\gamma\left(\mathrm{cm}^{-1}\right)=1721\left(\mathrm{C}=\mathrm{O}\right.$ stretching). $\mathbf{C}_{25} \mathbf{H}_{35} \mathbf{O}_{3} \mathbf{N}$ (397.55); Anal. Calc. (\%): C, 75.52; H, 8.89; N, 3.52. Found (\%): C, 75.35; H, 9.04; N, 3.13.

\section{(S)-4-(2-Methylbutoxy)phenyl 6-(4-dodecyloxyphenyl)pyridine-3-carboxylate (7a)}

Yield: $57 \%$; colorless crystals. ${ }^{1} \mathbf{H}$ NMR $\left(500 \mathrm{MHz} \mathrm{CDCl}_{3}\right): \delta(\mathrm{ppm})=9.41(\mathrm{~d}, J \approx 2.0 \mathrm{~Hz}$, $1 \mathrm{H}, \mathrm{Ar}-\mathrm{H}), 8.48\left(\mathrm{dd}, J_{1} \approx 8.3 \mathrm{~Hz}, J_{2}=2.0 \mathrm{~Hz}, 1 \mathrm{H}, \mathrm{Ar}-\mathrm{CH}\right), 8.10(\mathrm{~d}, J \approx 8.5 \mathrm{~Hz}, 2 \mathrm{H}, \mathrm{Ar}-\mathrm{CH})$, $7.84(\mathrm{~d}, J \approx 8.3 \mathrm{~Hz}, 1 \mathrm{H}, \mathrm{Ar}-\mathrm{CH}), 7.16(\mathrm{~d}, J \approx 9.0 \mathrm{~Hz}, 2 \mathrm{H}, \mathrm{Ar}-\mathrm{CH}), 7.05(\mathrm{~d}, J \approx 8.5 \mathrm{~Hz}, 2 \mathrm{H}, \mathrm{Ar}-$ $\mathrm{CH}), 6.97(\mathrm{~d}, J \approx 9.0 \mathrm{~Hz}, 2 \mathrm{H}, \mathrm{Ar}-\mathrm{CH}), 4.06\left(\mathrm{t}, J \approx 6.6 \mathrm{~Hz}, 2 \mathrm{H}, \mathrm{OCH}_{2}\right), 3.85-3.77(\mathrm{~m}, 2 \mathrm{H}$, $\left.\mathrm{OCH}_{2}\right), 1.93-1.88(\mathrm{~m}, 1 \mathrm{H}, \mathrm{CH}), 1.87-1.82\left(\mathrm{~m}, 2 \mathrm{H}, \mathrm{CH}_{2}\right), 1.65-1.29\left(\mathrm{~m}, 20 \mathrm{H}, \mathrm{CH}_{2}\right), 1.05(\mathrm{~d}$, $\left.J \approx 6.8 \mathrm{~Hz}, 3 \mathrm{H}, \mathrm{CH}_{3}\right), 0.98\left(\mathrm{t}, J \approx 6.5 \mathrm{~Hz}, 3 \mathrm{H}, \mathrm{CH}_{3}\right), 0.91\left(\mathrm{t}, J \approx 6.5 \mathrm{~Hz}, 3 \mathrm{H}, \mathrm{CH}_{3}\right) .{ }^{\mathbf{1 3}} \mathbf{C} \mathbf{~ N M R}$ $\left(125 \mathrm{MHz}, \mathrm{CDCl}_{3}\right): \delta(\mathrm{ppm})=164.51(\mathrm{COO}), 161.10,161.01,157.25,143.90,130.36,122.96$ $(\mathrm{Ar}-\mathrm{C}), 151.36,138.27,128.88,122.31,118.98,115.17,114.89(\mathrm{Ar}-\mathrm{CH}), 73.32,68.21$ 
$\left(\mathrm{OCH}_{2}\right), 34.73(\mathrm{CH}), 31.93,29.67,29.65,29.61,29.59,29.41,29.36,29.22,26.14,26.03$, $22.70\left(\mathrm{CH}_{2}\right), 16.52,14.13,11.33\left(\mathrm{CH}_{3}\right)$. FT-IR: $\gamma\left(\mathrm{cm}^{-1}\right)=1731(\mathrm{C}=\mathrm{O}$ stretching $)$. $\mathbf{C}_{35} \mathbf{H}_{47} \mathbf{O}_{4} \mathbf{N}$ (545.75); Anal. Calc. (\%): C, 77.02; H, 8.70; N, 2.56. Found (\%): C, 76.71; H, $8.37 ; \mathrm{N}, 2.24$.

\section{(S)-4-(3,7-Dimethyloctyloxy)phenyl 6-(4-dodecyloxyphenyl)pyridine-3-carboxylate (7b)}

Yield: $36 \%$; colorless crystals. ${ }^{1} \mathbf{H}$ NMR $\left(500 \mathrm{MHz} \mathrm{CDCl}_{3}\right): \delta(\mathrm{ppm})=9.32(\mathrm{~d}, J \approx 2.2 \mathrm{~Hz}$, $1 \mathrm{H}, \mathrm{Ar}-\mathrm{CH}), 8.37\left(\mathrm{dd}, J_{1} \approx 8.4, J_{2} \approx 2.2 \mathrm{~Hz}, 1 \mathrm{H}, \mathrm{Ar}-\mathrm{CH}\right), 7.99$ (d, $\left.J \approx 8.9 \mathrm{~Hz}, 2 \mathrm{H} \mathrm{Ar}-\mathrm{CH}\right)$, $7.74(\mathrm{~d}, J \approx 8.4 \mathrm{~Hz}, 1 \mathrm{H}, \mathrm{Ar}-\mathrm{CH}), 7.07$ (d, $J \approx 8.9 \mathrm{~Hz}, 2 \mathrm{H}, \mathrm{Ar}-\mathrm{CH}), 6.95(\mathrm{~d}, J \approx 8.9 \mathrm{~Hz}, 2 \mathrm{H}, \mathrm{Ar}-$ $\mathrm{CH}), 6.87(\mathrm{~d}, J \approx 8.9 \mathrm{~Hz}, 2 \mathrm{H}, \mathrm{Ar}-\mathrm{CH}), 3.96-3.93\left(\mathrm{~m}, 4 \mathrm{H}, \mathrm{OCH}_{2}\right), 1.78-1.73(\mathrm{~m}, 1 \mathrm{H}, \mathrm{CH})$, 1.55-1.46 (m, 3H, CH, CH $), 1.40-1.07\left(\mathrm{~m}, 26 \mathrm{H}, 13 \mathrm{CH}_{2}\right), 0.88$ (d, $\left.J \approx 6.6 \mathrm{~Hz}, 3 \mathrm{H}, \mathrm{CH}_{3}\right), 0.81$ $\left(\mathrm{m}, 9 \mathrm{H}, 3 \mathrm{CH}_{3}\right) .{ }^{13} \mathbf{C}$ NMR $\left(125 \mathrm{MHz}, \mathrm{CDCl}_{3}\right): \delta(\mathrm{ppm})=164.51(\mathrm{COO}), 161.08,161.04$, 157.05, 143.93, 130.41, 122.94 (Ar-C), 151.39, 138.24, 128.87, 122.33, 118.96, 115.16, 114.88 (Ar- $\mathrm{CH}), 68.20,66.77\left(\mathrm{OCH}_{2}\right), 39.25,37.29,36.21,31.93,29.67,29.65,29.60,29.58$, 29.40, 29.36, 29.21, 26.03, 24.67, $22.72\left(\mathrm{CH}_{2}\right), 29.85,27.98(\mathrm{CH}), 22.70,22.62,19.66,14.13$ $\left(\mathrm{CH}_{3}\right)$. FT-IR: $\gamma\left(\mathrm{cm}^{-1}\right)=1731\left(\mathrm{C}=\mathrm{O}\right.$ stretching). $\mathbf{C}_{\mathbf{4 0}} \mathbf{H}_{57} \mathbf{O}_{\mathbf{4}} \mathbf{N}$ (615.88); Anal. Calc. (\%): $\mathrm{C}$, 78.00; H, 9.35; N, 2.27. Found (\%): C, 77.67; H, 9.02; N, 1.89.

\section{(S)-[4-(2-Methylbutoxy)phenoxycarbonyl]phenyl}

\section{5-(4-dodecyloxyphenyl)pyridine-2-} carboxylate (10a)

Yield: $20 \%$; colorless crystals. ${ }^{\mathbf{1}} \mathbf{H}$ NMR $\left(500 \mathrm{MHz}_{\mathrm{CDCl}}\right): \delta(\mathrm{ppm})=8.98(\mathrm{~d}, J \approx 2.1 \mathrm{~Hz}$, 1H, Ar-CH), 8.29-8.20 (m, 3H, Ar-CH), 7.99 (dd, $\left.J_{1} \approx 7.8 \mathrm{~Hz}, J_{2} \approx 2.1 \mathrm{~Hz}, 1 \mathrm{H}, \mathrm{Ar}-\mathrm{CH}\right), 7.55$ $(\mathrm{d}, J \approx 7.8 \mathrm{~Hz}, 2 \mathrm{H}, \mathrm{Ar}-\mathrm{CH}), 7.36(\mathrm{~d}, J \approx 8.4 \mathrm{~Hz}, 2 \mathrm{H}, \mathrm{Ar}-\mathrm{CH}), 7.05(\mathrm{~d}, J \approx 8.4 \mathrm{~Hz}, 2 \mathrm{H}, \mathrm{Ar}-\mathrm{CH})$, $6.98(\mathrm{~d}, J \approx 7.8 \mathrm{~Hz}, 2 \mathrm{H}, \mathrm{Ar}-\mathrm{CH}), 6.87(\mathrm{~d}, J \approx 8.4 \mathrm{~Hz}, 2 \mathrm{H}, \mathrm{Ar}-\mathrm{CH}), 3.96(\mathrm{t}, J \approx 6.6 \mathrm{~Hz}, 2 \mathrm{H}$, $\left.\mathrm{OCH}_{2}\right), 3.76-3.67\left(2 \mathrm{~m}, 2 \mathrm{H}, \mathrm{OCH}_{2}\right), 1.85-1.72(\mathrm{~m}, 1 \mathrm{H}, \mathrm{CH}), 1.56-1.43\left(\mathrm{~m}, 2 \mathrm{H}, \mathrm{CH}_{2}\right), 1.40-$ $1.22\left(\mathrm{~m}, 20 \mathrm{H}, \mathrm{CH}_{2}\right), 0.95\left(\mathrm{~d}, J \approx 6.8 \mathrm{~Hz}, 3 \mathrm{H}, \mathrm{CH}_{3}\right), 0.89\left(\mathrm{t}, J \approx 7.5 \mathrm{~Hz}, 3 \mathrm{H}, \mathrm{CH}_{3}\right), 0.81(\mathrm{t}, J \approx$ $\left.6.7 \mathrm{~Hz}, 3 \mathrm{H}, \mathrm{CH}_{3}\right) .{ }^{13} \mathrm{C}$ NMR $\left(125 \mathrm{MHz}, \mathrm{CDCl}_{3}\right): \delta(\mathrm{ppm})=164.82,163.41(\mathrm{COO}), 160.32$, 157.16, 155.07, 144.87, 144.13, 140.44, 133.35, 127.56 (Ar-C), 134.49, 131.87, 127.66, 126.22, 122.36, 122.13, 115.44, 115.16, $114.74(\mathrm{Ar}-\mathrm{CH}), 73.31,68.28\left(\mathrm{OCH}_{2}\right)$ 34.73, 31.94, 29.69, 29.66, 29.63, 29.60, 29.41, 29.38, 29.21, 26.15, $26.05\left(\mathrm{CH}_{2}\right), 22.72,16.55,14.15$, $11.35\left(\mathrm{CH}_{3}\right)$. FT-IR: $\gamma\left(\mathrm{cm}^{-1}\right)=1751$ and $1735\left(2 \mathrm{C}=\mathrm{O}\right.$ stretching). $\mathbf{C}_{42} \mathbf{H}_{51} \mathbf{O}_{6} \mathbf{N}$ (665.86); Anal. Calc. (\%): C, 75.75; H, 7.73; N, 2.10. Found (\%): C, 75.43; H, 7.42; N, 1.76. 
(S)-[4-(3,7-Dimethyloctyloxy)phenoxycarbonyl]phenyl 5-(4-dodecyloxyphenyl)pyridine2-carboxylate (10b)

Yield: $29 \%$; colorless crystals. ${ }^{1} \mathbf{H}$ NMR $\left(500 \mathrm{MHz} \mathrm{CDCl}_{3}\right): \delta(\mathrm{ppm})=9.41(\mathrm{~d}, J \approx 2.1 \mathrm{~Hz}$, $1 \mathrm{H}, \mathrm{Ar}-\mathrm{CH}), 8.46\left(\mathrm{~d}, J_{l} \approx 8.4 \mathrm{~Hz}, J_{2} \approx 2.1 \mathrm{~Hz}, 1 \mathrm{H}, \mathrm{Ar}-\mathrm{CH}\right), 8.31$ (d, $\left.J \approx 8.6 \mathrm{~Hz}, 2 \mathrm{H}, \mathrm{Ar}-\mathrm{CH}\right)$, $8.08(\mathrm{dd}, J \approx 8.4 \mathrm{~Hz}, 1 \mathrm{H}$, Ar-CH) $7.84(\mathrm{~d}, J \approx 8.4 \mathrm{~Hz}, 2 \mathrm{H}, \mathrm{Ar}-\mathrm{CH}), 7.41(\mathrm{~d}, J \approx 8.6 \mathrm{~Hz}, 2 \mathrm{H}$, $\operatorname{Ar}-\mathrm{CH}), 7.13(\mathrm{~d}, J \approx 8.9 \mathrm{~Hz}, 2 \mathrm{H}, \mathrm{Ar}-\mathrm{CH}), 7.03(\mathrm{~d}, J \approx 8.4 \mathrm{~Hz}, 2 \mathrm{H}, \mathrm{Ar}-\mathrm{CH}), 6.95$ (d, $J \approx 8.9$ $\mathrm{Hz}, 2 \mathrm{H}, \mathrm{Ar}-\mathrm{CH}), 4.12-3.94$ (m, 4H, $\left.\mathrm{OCH}_{2}\right), 1.88-1.83$ (m, 1H, $\left.\mathrm{CH}\right), 1.54-1.47$ (m, 3H, CH, $\left.\mathrm{CH}_{2}\right), 1.44-1.15\left(\mathrm{~m}, 26 \mathrm{H}, \mathrm{CH}_{2}\right), 0.95\left(\mathrm{~d}, \mathrm{~J} \approx 6.6 \mathrm{~Hz}, 3 \mathrm{H}, \mathrm{CH}_{3}\right), 0.92-0.84\left(\mathrm{~m}, 9 \mathrm{H}, \mathrm{CH}_{3}\right) .{ }^{13} \mathbf{C}$ NMR (125 MHz, $\left.\mathrm{CDCl}_{3}\right): \delta(\mathrm{ppm})=164.75,163.35$ (COO), 160.32, 156.97, 155.08, 144.65, 144.20, 140.23, 128.36, 127.56 (Ar-C), 148.19, 134.43, 131.83, 128.57, 126.14, 122.34, 122.06, 115.44, 115.16 (Ar- $\mathrm{CH}), 68.27,66.78\left(\mathrm{OCH}_{2}\right), 39.25,37.29,36.22,31.91,29.64$, 29.62, 29.58, 29.57, 29.37, 29.33, 29.19, 26.02, 24.65, $22.70\left(\mathrm{CH}_{2}\right), 29.86,27.97(\mathrm{CH}), 22.67$, 22.60, 19.65, $14.10\left(\mathrm{CH}_{3}\right)$. FT-IR: $\gamma\left(\mathrm{cm}^{-1}\right)=1734$ (2 $\mathrm{C}=\mathrm{O}$ stretching). $\mathbf{C}_{\mathbf{4 7}} \mathbf{H}_{61} \mathbf{O}_{6} \mathbf{N}$ (735.99); Anal. Calc. (\%): C, 76.70; H, 8.37; N, 1.90. Found (\%): C, 76.41; H, 8.09; N, 2.19.

(S)-[4-(2-Methylbutoxy)phenoxycarbonyl]phenyl 6-(4-dodecyloxyphenyl)pyridine-3carboxylate (11a)

Yield: $33 \%$; colorless crystals. ${ }^{\mathbf{1}} \mathbf{H}$ NMR $\left(500 \mathrm{MHz}, \mathrm{CDCl}_{3}\right): \delta(\mathrm{ppm})=9.43(\mathrm{~d}, J \approx 2.1 \mathrm{~Hz}$, $1 \mathrm{H}, \mathrm{Ar}-\mathrm{CH}), 8.48\left(\mathrm{dd}, J_{1} \approx 8.4 \mathrm{~Hz}, J_{2} \approx 2.1 \mathrm{~Hz}, 1 \mathrm{H}, \mathrm{Ar}-\mathrm{CH}\right), 8.32(\mathrm{~d}, J \approx 8.5 \mathrm{~Hz}, 2 \mathrm{H}, \mathrm{Ar}-\mathrm{C} H)$, $8.10(\mathrm{~d}, J=8.5 \mathrm{~Hz}, 2 \mathrm{H}, \mathrm{Ar}-\mathrm{CH}), 7.86(\mathrm{~d}, J \approx 8.4 \mathrm{~Hz}, 1 \mathrm{H}, \mathrm{Ar}-\mathrm{CH}), 7.43(\mathrm{~d}, J \approx 8.5 \mathrm{~Hz}, 2 \mathrm{H}$, $\operatorname{Ar}-\mathrm{CH}), 7.15(\mathrm{~d}, J=8.5 \mathrm{~Hz}, 2 \mathrm{H}, \mathrm{Ar}-\mathrm{CH}), 7.05(\mathrm{~d}, J \approx 8.5 \mathrm{~Hz}, 2 \mathrm{H}, \mathrm{Ar}-\mathrm{CH}), 6.97$ (d, $J \approx 8.5$ $\mathrm{Hz}, 2 \mathrm{H}, \mathrm{Ar}-\mathrm{CH}), 4.07$ (t, $\left.J \approx 6.6 \mathrm{~Hz}, 2 \mathrm{H}, \mathrm{OCH}_{2}\right), 3.85-3.77\left(\mathrm{~m}, 2 \mathrm{H}, \mathrm{OCH}_{2}\right), 1.95-1.88(\mathrm{~m}, 1 \mathrm{H}$, $\mathrm{CH}), 1.89-1.78\left(\mathrm{~m}, 2 \mathrm{H}, \mathrm{CH}_{2}\right), 1.63-1.31\left(\mathrm{~m}, 20 \mathrm{H}, \mathrm{CH}_{2}\right), 1.05\left(\mathrm{~d}, J \approx 6.5 \mathrm{~Hz}, 3 \mathrm{H}, \mathrm{CH}_{3}\right), 0.98$ $\left(\mathrm{t}, J \approx 6.8 \mathrm{~Hz}, 3 \mathrm{H}, \mathrm{CH}_{3}\right), 0.91\left(\mathrm{t}, J \approx 6.8 \mathrm{~Hz}, 3 \mathrm{H}, \mathrm{CH}_{3}\right) .{ }^{13} \mathbf{C ~ N M R}\left(125 \mathrm{MHz}, \mathrm{CDCl}_{3}\right): \delta(\mathrm{ppm})$ $=164.79,163.56$ (COO), 161.36, 161.29, 157.18, 154.71, 144.11, 130.13, 129.00, 127.54 (Ar-C), 151.43, 138.45, 131.89, 130.05, 122.35, 121.95, 119.10, 115.16, 114.95 (Ar-CH), 73.32, $68.24\left(\mathrm{OCH}_{2}\right), 34.74(\mathrm{CH}), 31.94,29.69,29.66,29.63,29.60,29.42,29.38,29.22$, 26.15, 26.05, $22.72\left(\mathrm{CH}_{2}\right), 16.56,14.16,11.35\left(\mathrm{CH}_{3}\right)$. FT-IR: $\gamma\left(\mathrm{cm}^{-1}\right)=1732(2 \mathrm{C}=\mathrm{O}$ stretching). $\mathbf{C}_{42} \mathbf{H}_{51} \mathbf{O}_{6} \mathbf{N}$ (665.86); Anal. Calc. (\%): C, 75.75; H, 7.73; N, 2.10. Found (\%): C, 75.47; H, 7.38; N, 1.81. MS (ESI) (+): m/z (\%) = $666(100)\left[\mathrm{M}^{+}\right], 498(81)\left[\mathrm{M}^{+}-\mathrm{C}_{12} \mathrm{H}_{25}\right], 366$ (70) $\left[\mathrm{M}^{+}-\mathrm{C}_{18} \mathrm{H}_{19} \mathrm{O}_{4}\right]$. 
(S)-[4-(3,7-Dimethyloctyloxy)phenoxycarbonyl]phenyl 6-(4-dodecyloxyphenyl)pyridine3-carboxylate (11b)

Yield: $19 \%$; colorless crystals. ${ }^{1} \mathbf{H}$ NMR $\left(500 \mathrm{MHz}_{\mathrm{CDCl}}\right): \delta(\mathrm{ppm})=9.40(\mathrm{~d}, J \approx 2.1 \mathrm{~Hz}$, $1 \mathrm{H}, \mathrm{Ar}-\mathrm{CH}, 8.46\left(\mathrm{dd}, J_{l} \approx 8.4, \mathrm{~Hz}, J_{2} \approx 2.1 \mathrm{~Hz}, 1 \mathrm{H}, \mathrm{Ar}-\mathrm{CH}\right), 8.31(\mathrm{~d}, J \approx 8.6 \mathrm{~Hz}, 2 \mathrm{H}, \mathrm{Ar}-$ $\mathrm{CH}), 8.07(\mathrm{~d}, J \approx 8.7 \mathrm{~Hz}, 2 \mathrm{H}, \operatorname{Ar}-\mathrm{C} H), 7.83(\mathrm{~d}, J \approx 8.4 \mathrm{~Hz}, 1 \mathrm{H}, \operatorname{Ar}-\mathrm{CH}), 7.41(\mathrm{~d}, J \approx 8.6 \mathrm{~Hz}$, 2H, Ar-CH), $7.13(\mathrm{~d}, J \approx 8.9 \mathrm{~Hz}, 2 \mathrm{H}, \mathrm{Ar}-\mathrm{CH}), 7.03(\mathrm{~d}, J \approx 8.7 \mathrm{~Hz}, 2 \mathrm{H}, \mathrm{Ar}-\mathrm{CH}), 6.95(\mathrm{~d}, J \approx$ $8.9 \mathrm{~Hz}, 2 \mathrm{H}, \mathrm{Ar}-\mathrm{CH}), 4.05-3.97$ (m, 4H, 2H, $\left.\mathrm{OCH}_{2}\right), 1.93-1.86(\mathrm{~m}, 1 \mathrm{H}, \mathrm{CH}), 1.84-1.62(\mathrm{~m}$, $\left.3 \mathrm{H}, \mathrm{CH}, \mathrm{CH}_{2}\right), 1.48-1.16\left(\mathrm{~m}, 26 \mathrm{H}, \mathrm{CH}_{2}\right), 0.95$ (d, $\left.\mathrm{J} \approx 6.5 \mathrm{~Hz}, 3 \mathrm{H}, \mathrm{CH}_{3}\right), 0.89-0.78(\mathrm{~m}, 9 \mathrm{H}$, $\left.\mathrm{CH}_{3}\right) .{ }^{13} \mathrm{C}$ NMR $\left(125 \mathrm{MHz}, \mathrm{CDCl}_{3}\right): \delta(\mathrm{ppm})=164.77,163.60$ (COO), 161.43, 161.21, 156.96, 154.70, 144.11, 130.21. 127.49, 122.34 (Ar-C), 151.52, 138.34, 131.87, 128.94, 122.29, 121.94, 119.02, 115.12, 114.89 (Ar- $\mathrm{CH}), 68.20,66.73\left(\mathrm{OCH}_{2}\right), 39.24,37.28,36.20$, 31.92 , 29.67, 29.64, 26.60, 29.58, 29.40, 29.36, 29.20, 26.02, 24.66, $22.73\left(\mathrm{CH}_{2}\right), 29.83$, $27.98(\mathrm{CH}), 22.70,22.61,19.65,14.14\left(\mathrm{CH}_{3}\right)$. FT-IR: $\gamma\left(\mathrm{cm}^{-1}\right)=1734(2 \mathrm{C}=\mathrm{O}$ stretching $)$. $\mathbf{C}_{47} \mathbf{H}_{61} \mathbf{O}_{6} \mathbf{N}$ (735.99); Anal. Calc. (\%): C, 76.70; H, 8.37; N, 1.90. Found (\%): C, 76.40; H, 8.54; N, 1.89. MS (ESI) (+): $\mathrm{m} / \mathrm{z}(\%)=736(100)\left[\mathrm{M}^{+}\right], 366(35)\left[\mathrm{M}^{+}-\mathrm{C}_{23} \mathrm{H}_{29} \mathrm{O}_{4}\right], 198(20)$ $\left[\mathrm{C}_{12} \mathrm{H}_{7} \mathrm{NO}_{2}\right]$.

\section{Results and Discussions}

\subsection{Liquid crystalline properties}

The mesomorphic properties of the pyridine-based chiral calamitic compounds $4^{24}, \mathbf{5}, \mathbf{7 a}, \mathbf{b}$, 10a,b and 11a,b were investigated by using optical polarizing microscope (POM) and differential scanning calorimeter (DSC). The phase transitions temperatures observed on heating and cooling cycles and corresponding transition enthalpies of the compounds are given in Table 1. The peak temperatures are given in degree Celsius and the numbers in parentheses indicate the transition enthalpy $(\Delta H)$.

DSC and POM analyses have revealed that the nicotinate ester 5 compound displays only a monotropic SmA phase upon cooling from the isotropic state (see Table 1, Fig. 1). The DSC traces displays two endotherms upon heating associated to a $\mathrm{Cr}_{1}-\mathrm{Cr}_{2}$-Iso phase sequence and two exotherms upon cooling associated to the phase sequence Iso-(SmA)- $\mathrm{Cr}_{1}$. On the contrary, the methyl picolinate ester 4 , which was previously reported, ${ }^{24}$ exhibits enantiotropic smectic A mesophase. The mesophase of both simple pyridine-based methyl esters $\mathbf{4}$ and $\mathbf{5}$ carrying a $n$ dodecyloxy chain is characterized by the typical fan-shaped texture (see Fig. 2a,b). The comparison of the nicotinate-based compound 5 with the picolinate-based compound $\mathbf{4}^{24}$, 
shows that the position of the nitrogen does not affect the type of the mesophase but increases the mesophase stability. A stable molecular stacking is possible with the intra-molecular interactions of nitrogen atom placed at the ortho position of the ester group instead of inner position.

Table 1 Mesophase, phase transition temperatures $\left({ }^{\circ} \mathrm{C}\right)$ and the corresponding transition enthalpies $(\Delta H \mathrm{~kJ} / \mathrm{mol})^{\mathrm{a}}$ of the compounds $4^{24}, \mathbf{5}, \mathbf{7 a}, \mathbf{b}, \mathbf{1 0 a}, \mathbf{b}$ and 11a,b.

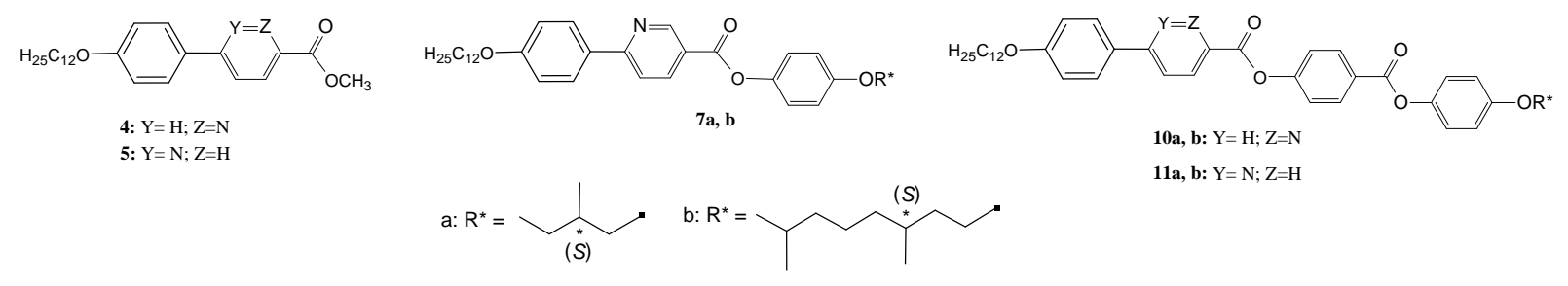

\begin{tabular}{lll}
\hline Heating & Cooling \\
\hline $\mathbf{4}^{\mathbf{2 4}}$ & $\mathrm{Cr} 105.90[48.22] \mathrm{SmA} 118.98$ [5.14] Iso & Iso 114.90 [6.22] SmA 91.36 [52.65] Cr
\end{tabular}

\begin{tabular}{lll}
\hline 5 & $\mathrm{Cr}_{1} 119.55[3.89] \mathrm{Cr}_{2}$ 126.01 [32.63] Iso & Iso 117.26 [2.36] (SmA) 113.66 [49.88] \\
& $\mathrm{Cr}_{1}$
\end{tabular}

\begin{tabular}{lll}
\hline 7a & Cr 102.80 [38.63] SmC*161[XX] SmA & Iso 170.23 [3.43] SmA 165 [XX] SmC* \\
& 173.27 [3.64] Iso & $83.35[39.55] \mathrm{Cr}$ \\
\hline 7b & Cr 94.56 [26.38] SmC* $143^{\mathrm{b}} \mathrm{SmA} \mathrm{154.14}$ & Iso 150.78 [2.77] SmA 147 ${ }^{\mathrm{b}} \mathrm{SmC}^{*} 65.44$ \\
& [2.74] Iso & {$[24.74] \mathrm{Cr}$}
\end{tabular}

10a $\quad$ Cr $112^{\mathrm{b}} \mathrm{M}_{1} 215^{\mathrm{b}}$ decomp. Decomposed sample after $1^{\text {st }}$ heating.

$\mathbf{1 0 b}^{\mathrm{c}} \mathrm{Cr}_{1} 65.33$ [8.22] $\mathrm{Cr}_{2} 121.10$ [37.35] $\mathrm{M}_{1} \quad$ Decomposed sample after $1^{\text {st }}$ heating. $228.77[9.32]$ decomp.

\begin{tabular}{lll}
\hline 11a & $\mathrm{Cr}_{1} 112.65$ [29.51] $\mathrm{Cr}_{2} 122.89[10.17]$ & Iso 270.09 [2.71] $\mathrm{SmA} 234^{\mathrm{b}} \mathrm{SmC}^{*} 91.72$ \\
& $\mathrm{SmC}^{*} 248^{\mathrm{b}} \mathrm{SmA} 273.85[3.30]$ Iso & {$[24.75] \mathrm{Cr}_{1}$} \\
\hline 11b & $\mathrm{Cr}_{1} 104.46[30.88] \mathrm{Cr}_{2} \mathrm{XX}[\mathrm{XX}] \mathrm{SmC}^{*}$ & Iso 234.52 [3.61] SmA 222.21 SmC* XX \\
& $224.59 \mathrm{SmA} 236.97$ [3.92] Iso & {$[\mathrm{XX}] \mathrm{Cr}_{2} 82.99$ [32.92] Cr }
\end{tabular}

\footnotetext{
${ }^{\text {a }}$ Perkin-Elmer DSC-6; enthalpy values in italics in brackets taken from the $2^{\text {nd }}$ heating and cooling scans at a rate of $10{ }^{\circ} \mathrm{C} \mathrm{min}{ }^{-1}$; Abbreviations: $\mathrm{Cr}=$ crystalline, $\mathrm{SmA}=$ smectic $\mathrm{A}$ mesophase, $\mathrm{SmC}^{*}=$ chiral smectic $\mathrm{C}$ phase, $\mathrm{M}_{1}=$ uncharacteristic mesophase, Iso $=$ isotropic
} 
liquid phase. ${ }^{\mathrm{b}}$ These transitions were determined by POM. ${ }^{\mathrm{c}} 1^{\text {st }}$ heating scan at a rate of $10{ }^{\circ} \mathrm{C}$ $\min ^{-1}$ was only carried out due to decomposition.
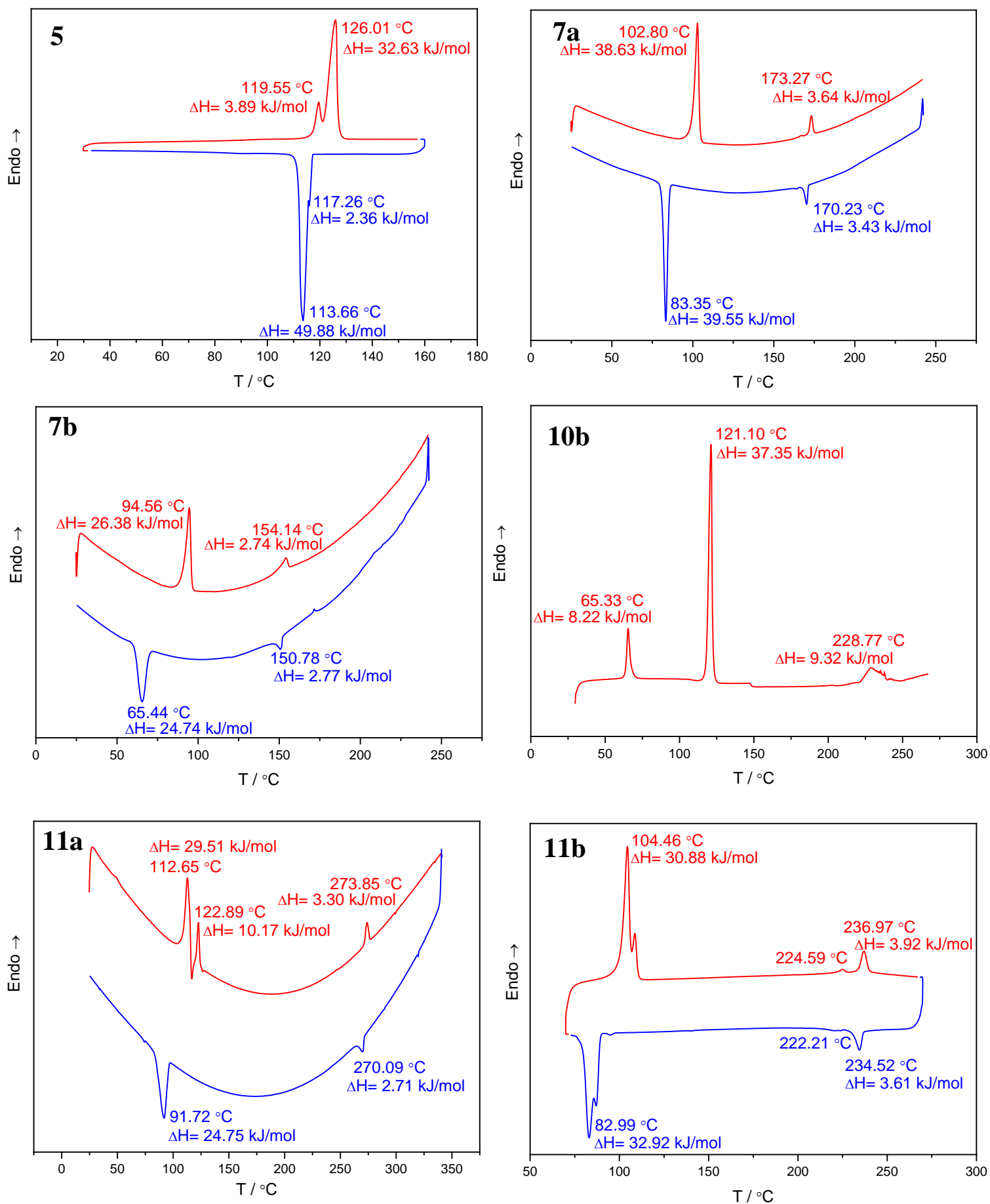

Fig. 1. DSC thermograms of compounds $\mathbf{5}, \mathbf{7 a}, \mathbf{b}$ and $\mathbf{1 1 a}, \mathbf{b}$ on $2^{\text {nd }}$ heating and cooling and DSC thermogram of $\mathbf{1 0 b}$ on $1^{\text {st }}$ heating $\left(10^{\circ} \mathrm{C} \cdot \mathrm{min}^{-1}\right)$. 

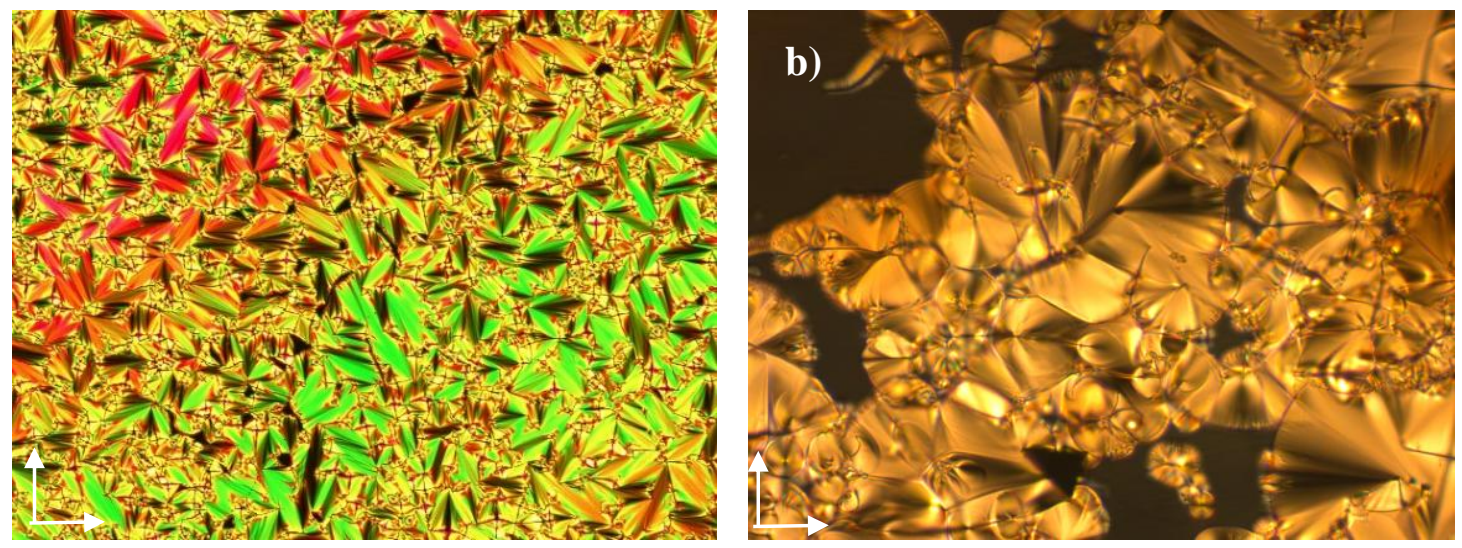

Fig. 2. Optical textures of SmA mesophase as observed between crossed polarizers (indicated by arrows) between ordinary glass plates on cooling; (a) fan-shaped texture of compound 4 at $\mathrm{T}=111^{\circ} \mathrm{C}$; (b) fan-shaped texture of compound $\mathbf{5}$ obtained at $\mathrm{T}=113{ }^{\circ} \mathrm{C}$.

The DSC thermograms of compound 7a carrying a (S)-2-methylbutyloxy end chain shows three endotherms for a phase transition sequence of crystal $(\mathrm{Cr})$-chiral smectic $\mathrm{C}^{*}\left(\mathrm{SmC}^{*}\right)$ smectic A (SmA) - isotropic phase (Iso) and three exotherms for the reverse phase transition sequence (Table 1, Fig. 1). An enantiotropic chiral tilted mesophase (SmC*) with a Schlieren texture with some striped regions was clearly (Fig. 3a). Additionally, SmA phase appeared with a homeotropic texture between the $\mathrm{SmC}^{*}$ phase and the isotropic phase (Fig. 3b).

Compound $\mathbf{7 b}$ terminated with a $(S)$-3,7-dimethyloctyloxy chiral chain, which is a longer and more branched chain, shows $\mathrm{SmC}^{*}$ and SmA mesophases with at lower transition temperatures compared to compound 7a (Table 1). Compound 7b showed only two endotherms and two exotherms corresponding to $\mathrm{Cr}$ - SmC* and SmA - Iso transitions on the DSC heating and cooling traces. The SmA - SmC* phase transition is not detectable by on the DSC thermogram but is clearly evidenced by POM observations (see Fig. 3c,d). It can be noticed that the increase in chain length and branching give rise to a decrease of the phase transition temperatures as well as the temperature range of chiral tilted mesophase.

The four-ring calamitic nicotinate-based compound 10a and 10b exhibits a mesophase on heating. However, both compounds 10a and 10b decompose around $215^{\circ} \mathrm{C}$ and $228{ }^{\circ} \mathrm{C}$, respectively. For compound $\mathbf{1 0 a}$, due to decomposition, the mesophase was assigned as uncharacteristic mesophase $\mathrm{M}_{1}$ (see Fig.24, ESI). The DSC heating curve of compound 10b 
displays to clear endotherms centered at 65 and $121^{\circ} \mathrm{C}$ attributed to a crystal to crystal phase transition and to a crystal to an uncharacteristic $\mathrm{M}_{1}$ mesophase. A third endotherm, associated to the decomposition of the compound, is observed around $218{ }^{\circ} \mathrm{C}$. No exotherm could be detected after decomposition during the cooling process (Fig. 1).

Interestingly, the four-ring calamitic picolinate-based compounds 11a and 11b exhibit a more stable mesomorphism as compared to compounds $\mathbf{1 0 a}$ and $\mathbf{1 0 b}$. The DSC thermograms of $11 \mathbf{a}$ show three endotherms which corresponds to $\mathrm{Cr}_{1} \rightarrow \mathrm{Cr}_{2}, \mathrm{Cr}_{2} \rightarrow \mathrm{SmC}^{*}$ and $\mathrm{SmA} \rightarrow$ Iso phase transition. The exotherm associated to the SmC*-SmA phase transition was not observed on the heating curve. However, POM observations clearly revealed that when the temperature reaches $122^{\circ} \mathrm{C}$, an characteristic texture associated to a SmC* mesophase readily develops and when the temperature reaches $248{ }^{\circ} \mathrm{C}$, the texture transforms into a characteristic SmA fan-shaped texture (Fig. 3e,f). On cooling, two exotherms associated to Iso-SmA and SmC*$\mathrm{Cr}_{1}$ phase transition were observed and similarly the $\mathrm{SmA} \rightarrow \mathrm{SmC}^{*}$ transition was only detected at $234{ }^{\circ} \mathrm{C}$ by POM. For compound 11b carrying a (S)-3,7-dimethyloctyloxy chiral chain, four endotherms and four exotherms are observed on the DSC traces and correspond to a reversible $\mathrm{Cr}_{1}-\mathrm{Cr}_{2}-\mathrm{SmC}^{*}$-SmA-Iso phase transition sequence. Typical finger-print texture of $\mathrm{SmC}^{*}$ and fan-shaped texture of SmA phase observed for compounds $\mathbf{1 1 b}$ are shown in Fig. $3 \mathrm{~g}, \mathrm{~h}$.

In comparison, the corresponding biphenyl analogues present a richer polymorphism ${ }^{27}$ with at least three different mesophases (SmC, SmA and higher ordered smectic phase). In reported series here, we can notice that the clearing point is drastically increasing with the number of rings within the core. Indeed, mesophase-to-isotropic transition temperature increases up to $273^{\circ} \mathrm{C}$ for compound 11a. Additionally, an increase in the mesomorphic range was observed when the number of aromatic cores is increased.

The position of the nitrogen in the aromatic ring gives rise to drastic effects on the mesomorphic properties. Comparison of the pyridine-based methyl esters $\mathbf{4}$ and $\mathbf{5}$ compounds, which differ only by the position of the nitrogen atom, shows that compound $\mathbf{5}$ present a monotropic smectic A phase over a very narrow mesomorphic range while $\mathbf{4}$ shows a welldefined enantiotropic SmA phase over a larger temperature range. Similarly, the picolinate based compound 10a and nicotinate based compound 11a, which both of them carrying a short chiral chain but differing only by the position of the nitrogen, an uncharacteristic mesophase, which decomposed at high temperatures, appeared for 10a whereas the nicotinate aromatic ring in compound 11a allows the formation of lamellar mesophases $\left(\mathrm{SmC}^{*}\right.$ and 
SmA) at high temperatures (see Fig. 3g,h) without decomposition. A similar behavior was observed with 10b and 11b, presenting a long chiral chain and differing only by the position of the nitrogen, the nicotinate core leads to the occurrence of more stable mesophases such as SmC* and SmA without a decomposition of sample at high temperatures. These results demonstrate that the position of the nitrogen in four-rings calamitic mesogens prevails on mesophase stability and leads to favor the emergence of a SmA mesophase, due to the increased lateral interactions, in line with the conclusions already reported by Chia et al. ${ }^{\text {Ereur : }}$ Signet non défini. The length and the branching of chiral end chain also impact the transition temperatures and the extent of the mesomophic range.
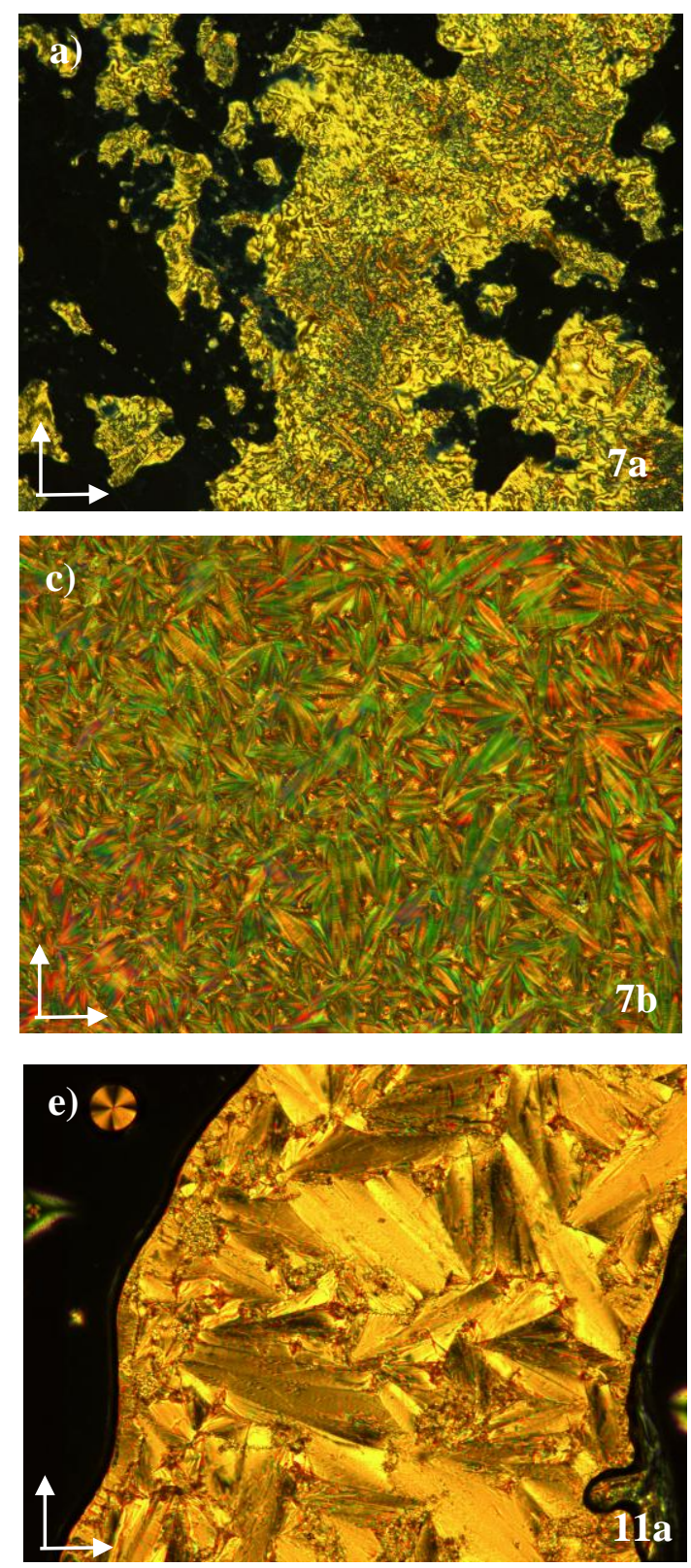

g)
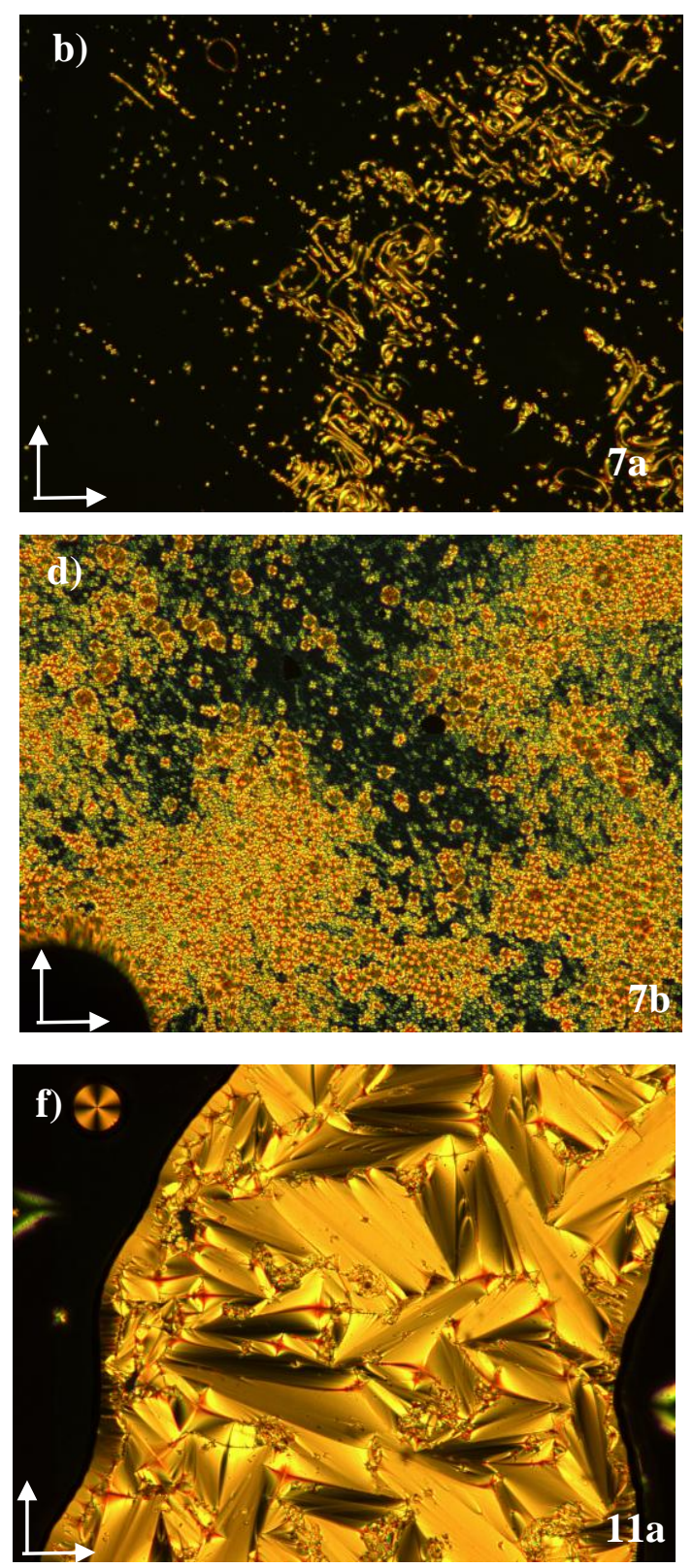

h)

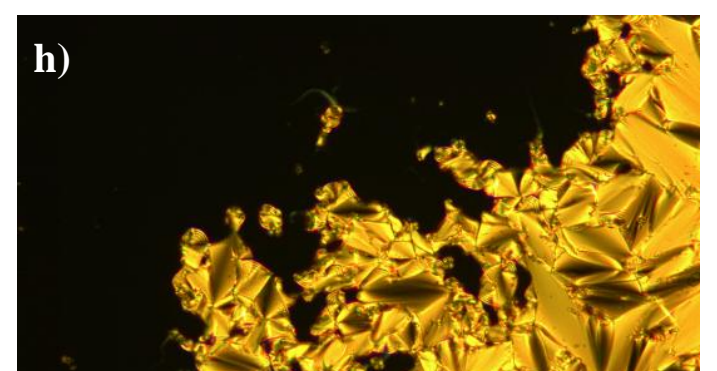


Fig. 3. Optical textures of the mesophases as observed between crossed polarizers (indicated by arrows) between ordinary glass plates; (a) Schlieren texture of the $\mathrm{SmC}^{*}$ mesophase at $\mathrm{T}=$ $165{ }^{\circ} \mathrm{C}$ and (b) homeotropic texture of the SmA mesophase at $\mathrm{T}=170{ }^{\circ} \mathrm{C}$ of compound $7 \mathbf{a}$ on cooling; (c) finger-print texture of the SmC* mesophase at $\mathrm{T}=140{ }^{\circ} \mathrm{C}$ on cooling and (d) focal-conic texture of the SmA mesophase at $\mathrm{T}=147{ }^{\circ} \mathrm{C}$ of compound $\mathbf{7 b}$ on heating; (e) texture characteristic of a SmC* mesophase at $\mathrm{T}=223^{\circ} \mathrm{C}$ and (f) fan-shaped SmA texture at $\mathrm{T}=257^{\circ} \mathrm{C}$ of compound 11a on cooling; (g) finger-print texture of the $\mathrm{SmC} *$ mesophase at $\mathrm{T}$ $=221{ }^{\circ} \mathrm{C}$ and $(\mathrm{h})$ fan-shaped $\mathrm{SmA}$ texture at $\mathrm{T}=232{ }^{\circ} \mathrm{C}$ of compound $\mathbf{1 1 b}$ on cooling.

To further confirmed, the mesophase formations and the phase assignments deduced for compounds 5, 7a,b and $\mathbf{1 1 b}$ by POM observations, temperature-dependent XRD analyses were also conducted. More information, about the distinct phases, derived from XRD are presented in the following section.

\subsection{SAXS Measurements}

The temperature-dependent SAXS patterns recorded on compound $\mathbf{5}$ confirm that two thermal transitions occur between $90-110{ }^{\circ} \mathrm{C}$ and between $125-130{ }^{\circ} \mathrm{C}$ upon heating (Fig. 4), agreeing with the transition temperatures detected by DSC. The diffractograms recorded below $125^{\circ} \mathrm{C}$ display several sharp peaks over the whole $2 \theta$ range explored, typical of a crystalline compound. Above $125^{\circ} \mathrm{C}$, the diffractograms present only two broad halos at 26.8 and $4.6 \AA$ associated to the mean distances between molecules in a disordered state. The low temperature transition is thus attributed to a crystal to crystal transformation whereas the high temperature transition is attributed to a crystal to isotropic phase transition. Upon cooling from the isotropic state, a first thermal transition is detected between $130{ }^{\circ} \mathrm{C}$ and $120{ }^{\circ} \mathrm{C}$ and is associated to a crystallization of the compound. In fact, the SAXS patterns recorded below $120{ }^{\circ} \mathrm{C}$, display several sharp peaks in the small and wide-angle regions (Fig. 4b). Another crystal to crystal phase transition has also been detected between $110{ }^{\circ} \mathrm{C}$ and $90{ }^{\circ} \mathrm{C}$. 
Transition temperature discrepancies can be observed between SAXS and DSC measurements due to experimental kinetic aspects. During the cooling, the formation of a monotropic Smectic A phase could not have been confirmed by SAXS measurements and only crystalline states have been detected below the isotropic phase. The observation by POM of the monotropic SmA phase is likely attributed to kinetic effects.

a)

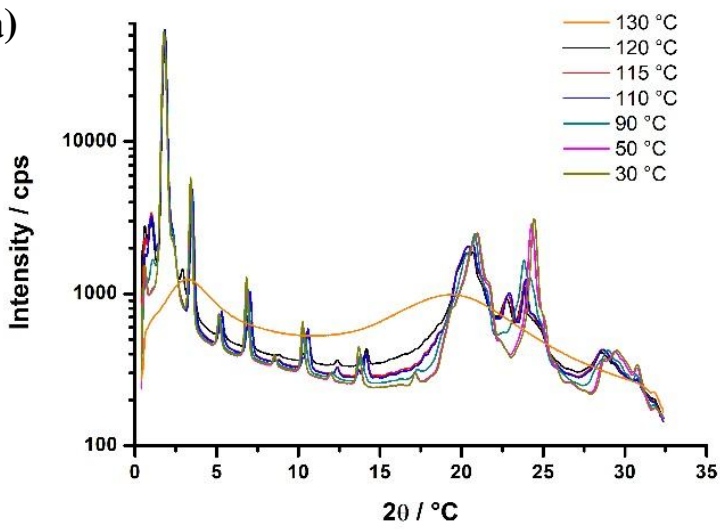

b)

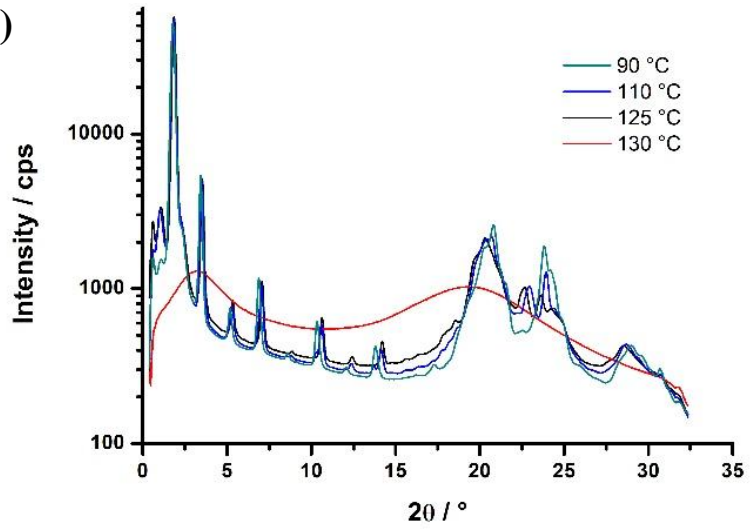

Fig. 4. SAXS diffractograms recorded of compound 5 upon heating (a) and cooling (b).

The diffraction patterns obtained as a function of temperature on compound 7a were first analyzed upon cooling (Fig. 5a). At $175^{\circ} \mathrm{C}$, the compound is an isotropic state in accordance with the POM observations and only two broad halos centered at 30.2 and $4.8 \AA$ are present on the SAXS pattern. Between, $170{ }^{\circ} \mathrm{C}$ and $110^{\circ} \mathrm{C}$, all the SAXS patterns display a sharp and intense (001) reflection together with a weak second order reflection (002). The broad halo centered around 4.7-4.5 $\AA$, confirm the liquid crystalline nature of the phase. The phase transition between the smectic A phase and the smectic $\mathrm{C}^{*}$ phase detected by POM observations was found difficult to detect by SAXS measurements since no marked change have been observed in the temperature range $170-165{ }^{\circ} \mathrm{C}$. The smectic $\mathrm{C}^{*}$ phase is certainly the most thermodynamically stable phase. Below $110{ }^{\circ} \mathrm{C}$, the compound is in a crystalline state. Upon heating, a phase transition from a crystalline state to a smectic mesophase and a phase transition from a smectic mesophase to the isotropic liquid are clearly detected around 90-110 ${ }^{\circ} \mathrm{C}$ and $170-175^{\circ} \mathrm{C}$, respectively, but, here also, the smectic $\mathrm{C}^{*}$ to smectic A phase transition could have be properly detected. The SAXS pattern recorded at $110{ }^{\circ} \mathrm{C}$ upon cooling is presented on Fig. 5b. The (001) reflection is centered at $31.3 \AA$ and the second 
order reflection at $15.7 \AA$. The interlayer distance measured is in accordance with a formation of monolayers of molecules. The decrease of the d-spacing upon cooling indicates the tilted organization of the molecules within the SmC* layers. From, this lamellar period and the length of the molecule ( $35.5 \AA$ in a fully extended conformation), a tilt angle value of $28^{\circ}$ can be estimated at $110^{\circ} \mathrm{C}$.
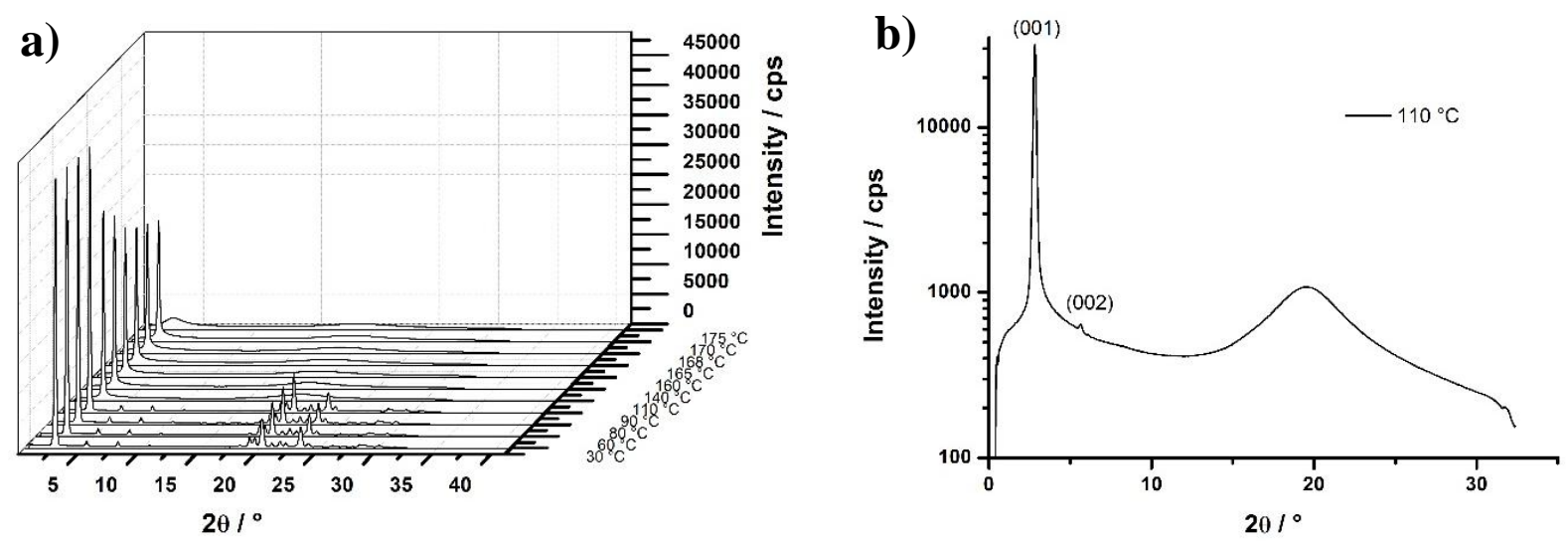

Fig. 5. a) SAXS patterns obtained as a function of the temperature with compound 7a upon cooling; b) SAXS pattern obtained in the smectic $\mathrm{C}^{*}$ phase of compound $7 \mathbf{a}$ at $110{ }^{\circ} \mathrm{C}$ upon cooling.

Analysis of the diffraction patterns obtained with compound $\mathbf{7 b}$ as a function of temperature upon heating and cooling confirms the presence of two reversible phase transition centered around $160{ }^{\circ} \mathrm{C}$ and $90{ }^{\circ} \mathrm{C}$ (Fig. 6). Here, also, the SmA-SmC* phase transition could not have been detected. Above $160{ }^{\circ} \mathrm{C}$, the compound is in anisotropic state and only broad halos, associated to mean intermolecular distances have been detected. Below, the $90{ }^{\circ} \mathrm{C}$, the compound $\mathbf{7 b}$ is a crystalline sate, as confirmed by the presence of numerous sharp diffractions peak in the wide and small angles regions. The SAXS patterns recorded between $90{ }^{\circ} \mathrm{C}$ and $160{ }^{\circ} \mathrm{C}$ confirm the formation of a liquid crystal state. Only one sharp reflection could have been detected in the small angle region but, based on the POM observations, this reflection can be indexed as the (001) reflection of a lamellar phase. The absence of higher order reflections indicates a less ordered arrangement of the molecules compare to the previous compound in the $\mathrm{SmC}^{*}$ mesophase. A broad peak, reflecting the fluidity of the phase, is also observed around $18.85^{\circ}$ in the wide angle region. The molecular length in the most extended form of the all-trans configuration of the molecule, measured using a Chem3D 
molecular model, is $39.5 \AA$. The interlayer distance measured at $150{ }^{\circ} \mathrm{C}$ is $34 \AA$, which much smaller than the molecular length, confirming that the molecules are tilted inside the monolayers. The tilt angle calculated at $150^{\circ} \mathrm{C}$, in the smectic $\mathrm{C}^{*}$ phase, is around $30.5^{\circ}$. The tilt angle shift from $29.5^{\circ}$ to $32^{\circ}$ upon cooling from $155^{\circ} \mathrm{C}$ to $100{ }^{\circ} \mathrm{C}$.

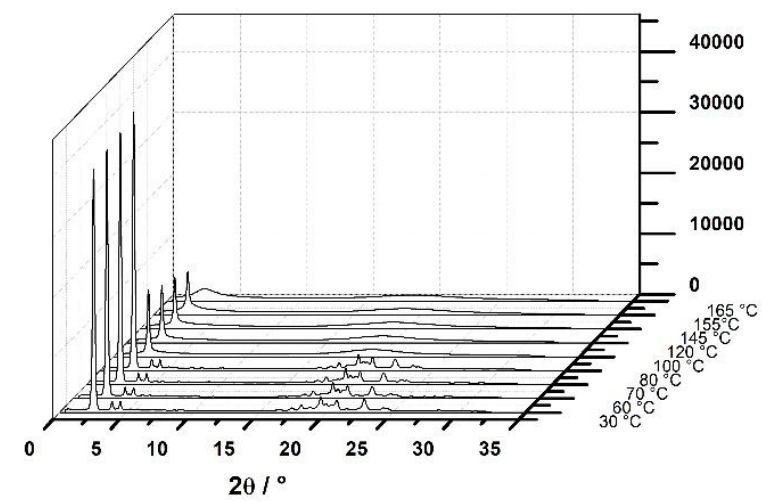

Fig. 6. SAXS patterns recorded on compound $7 \mathbf{b}$ upon cooling.

The temperature-dependent SAXS patterns confirm that compound 11b undergoes several reversible phase transitions (Fig. 7a). On heating, below $100{ }^{\circ} \mathrm{C}$, this compound is in a crystalline state as confirmed by the observations of numerous sharp diffraction peaks over the whole $2 \theta$ range explored. Above $120{ }^{\circ} \mathrm{C}$, the compound is clearly in a smectic liquid crystalline phase. Two sharp reflections, indexed as the (001) and (002) reflections of a lamellar phase, are observed in the small angles region as well as a broad peak around $19^{\circ}$ in $2 \theta$ in the wide angles region. By POM observation, this mesophase was identified as a smectic $C^{*}$ phase. The length of the molecule in a fully extended conformation is around $\sim 45$ $\AA$. So, the mesophase is constituted of monolayers of tilted molecules. The molecular tilt estimated at $140{ }^{\circ} \mathrm{C}$ from an interlayer distance of $39.5 \AA$ is around $28.5^{\circ}$ and this tilt decrease with the temperature increase (Fig. 7b). The molecules are likely arranged in head-to-tail fashion such as the space symmetry is broken into a phase of $\mathrm{C} 2$ symmetry. ${ }^{28}$ No information about the helical pitch could have been extracted from the SAXS patterns. Entering in the smectic A phase around $230{ }^{\circ} \mathrm{C}$, the intensity of the first order reflection strongly decrease and shift to higher distance, at a distance close to the molecular length $\mathrm{d} \sim 1$, and the second order reflection completely vanished. Finally, at $245^{\circ} \mathrm{C}$, the compound is in an isotropic state. The reverse sequence was observed upon cooling from the isotropic state and the phase transitions were detected in the same temperature range as determined by DSC. The $\mathrm{Cr}_{1}-\mathrm{Cr}_{2}$ 
and the $\mathrm{Cr}_{2}-\mathrm{SmC} *$ transitions were too close to properly isolate the SAXS pattern of the $\mathrm{Cr}_{2}$ phase.
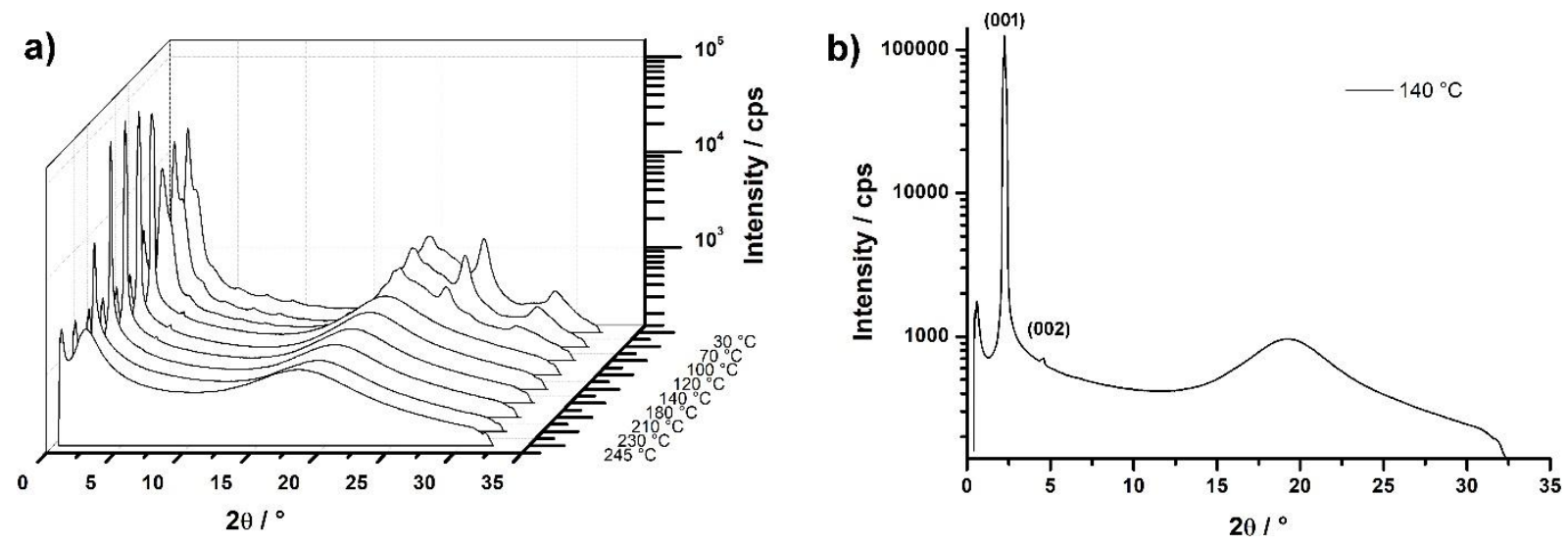

Fig. 7. a) Temperature-dependent SAXS patterns measured on compound 11b compound on heating; b) SAXS pattern measured at $140{ }^{\circ} \mathrm{C}$ on heating.

\section{Conclusion}

In this article, chiral multi-ring calamitic molecules with a specific architecture have been designed in order to examine structure-mesogenicity relationship and obtain new liquid crystal materials that may be useful for potential applications. The effects of the introduction of a heteroatom via nicotinate and picolinate based cores on the mesomorphic properties of calamitic compounds with a chiral end chain such as (S)-2-methylbutyloxy or (S)-3,7dimethyloctyloxy have been investigated by POM observation, DSC measurement and XRD analysis.

All the calamitic compounds exhibited liquid crystalline behaviour by forming ordered monolayer smectic phases depending on type of hetero aromatic rings, number of aromatic cores and the type of chiral branched chain. Firstly, the extension of the core via an increase of the number of aromatic rings leads to an increase of the mesophases stability and an expansion of the mesophase range. The length and branching of the chiral terminal chains play an effective role on transition temperatures and on the mesomorphic ranges. Additionally, it can be concluded that the inner position of the nitrogen in three or four-rings 
calamitic mesogens gives rise to more stable smectic phases due to enhanced lateral interactions.

\section{Acknowledgement}

This research has been supported by The Scientific and Technological Research Council of Turkey (TÜBITAK), Turkey with the Project Number 116Z465. The authors are grateful to the TÜBİTAK-2232 programme for a support with Project Number 118C273. F.C. and O.J. also thank the Institut des Sciences Chimiques de Rennes, the University of Rennes 1 and Rennes Metropole for their financial support.

\section{Appendix A. Supplementary data}

Electronic supplementary information (ESI) is available.

\section{References}

1 Tschierske C. Development of Structural Complexity by Liquid Crystal Self-assembly. Angew. Chem. Int. Ed. 2013;52:8828-8878.

${ }^{2}$ Kohout M, Bubnov A, Šturala J, Novotná V, Svoboda J. Effect of alkyl chain length in the terminal ester group on mesomorphic properties of new chiral lactic acid derivatives. Liq. Cryst. 2016;43:1472-1475.

${ }^{3}$ Lehmann M, Kestemont G, Aspe RG, Buess-Herman C, Koch MHJ, Debije MG, Piris J, De Haas MP, Warman JM, Watson MD, Lemaur V, Cornil J, Geerts YH, Gearba R, Ivanov DA. High Charge-Carrier Mobility in $\pi$-Deficient Discotic Mesogens: Design and StructureProperty Relationship. Chem. Eur. J. 2005;11:3349-3362.

${ }^{4}$ O'Neill M, Kelly SM. Liquid Crystals for Charge Transport, Luminescence, and Photonics. Adv. Mater. 2003;15:1135-1146.

${ }^{5}$ Girotto CE, Bechtold IH, Gallardo H. New liquid crystals derived from thiophene connected to the 1,2,4-oxadiazole heterocycle. Liq. Cryst. 2016;12:1768-1777.

${ }^{6}$ Ghosh T, Lehmann M. Recent Advances in Heterocycle-Based Metal-Free Calamitics. J. Mater. Chem. C 2017;5:12308-12337.

${ }^{7}$ Ong LK, Ha ST, Yeap GY, Lin HC. Heterocyclic pyridine-based liquid crystals: synthesis and mesomorphic properties. Liq. Cryst. 2018;45:1574-1584.

${ }^{8}$ Campbell NL, Duffy WL, Thomas GI, Wild JH, Kelly SM, Bartle K, O'Neill M, Minter V, Tuffin RP. Nematic 2,5-disubstituted thiophenes. J. Mater. Chem. 2002:12:2706-2721. 
${ }^{9}$ Burrow MP, Gray GW, Lacey D, Toyne KJ. The synthesis and liquid crystal properties of some 2,5-disubstituted pyridines. Liq. Cryst. 1988;3:1643-1653.

${ }^{10}$ Chia WL, Tsai CY. Synthesis and mesomorphic properties of a series of phenyl 6-(4alkoxyphenyl) nicotinates. Heterocycles 2011;83:1057-1065.

${ }^{11}$ Chia WL, Lin CW. Synthesis and thermotropic studies of a novel series of nematogenic liquid crystals 2-(6-alkoxynaphthalen-2-yl)-5-cyanopyridines. Liq. Cryst. 2013;40:922-931.

${ }^{12}$ Petrov VF. Nitrogen-containing fused heterocycles as the structural fragments in calamitic liquid crystals. Liq. Cryst. 2001;28:217-240.

${ }^{13}$ Nash JA, Gray GW. Studies of Some Heterocyclic Mesogens. Mol. Cryst. Liq. Cryst. 1974;25:299-321.

14 Rogojerov M, Jordanov B, Antonov L, Hinrichs K. Determination of the average orientation of 4-phenylpyridine in nematic solvent by means of infrared linear dichroism: Study of its conformational dependence on the dihedral angle between aromatic rings. J. Mol. Struct. 2008;875:540-548.

${ }^{15}$ Yu H, Szilvási T, Rai P, Twieg RJ, Mavrikakis M, Abbott NL. Computational ChemistryGuided Design of Selective Chemoresponsive Liquid Crystals Using Pyridine and Pyrimidine Functional Groups. Adv. Funct. Mater. 2018;28:q1703581-1703591.

${ }^{16}$ Chia WL, Huang HS. Effect of Pyridine on the Mesophase of Teraryl Liquid Crystals: A New Series of Nematic Liquid Crystals Named 2-(4-Alkoxybiphen-4'-yl)-5-methylpyridines. Int. J. Mol. Sci. 2016;17:344-358.

${ }^{17}$ Byron DJ, Lacey D, Wilson RC. Properties of the Liquid Crystals Formed by Certain 4-(2'Pyridyl)phenyl and 4- (4'-Pyridyl)phenyI 4"-n -Aikoxybenzoates. Mol. Cryst. Liq. Cryst. 1980;62:103-114.

${ }^{18}$ Kamogawa H, Kawashima K, Shimizu M, Sakakibara Y. Synthesis of mesomorphic aryl esters bearing a pyridine ring. Liq. Cryst. 1991;10:101-110.

${ }^{19}$ Nobuhiro O, Noriko Y, Ichiro K, Hitoshi S, Yasuhiro H. Preparation of optically active 4alkoxycarbonylphenyl 5-phenyl-3-pyridinecarboxylate derivatives as liquid crystals and antiferroelectric liquid crystals having tristable state. Jpn. Kokai Tokkyo Koho 1993;JP 05201983 A 19930810.

${ }^{20}$ Yuzo S, Norio K, Masami Y, Akira M. Optically active pyridine carboxylate derivatives as liquid crystals. Jpn. Kokai Tokkyo Koho 1989; JP 01019068 A 19890123. 
21 Yoshitake S, Shuhe Y. Optically active 3-phenylpyridine-2-carboxylate esters as $\begin{array}{llllll}\text { ferroelectric smectic liquid } & \text { crystals. Jpn. Kokai Tokkyo }\end{array}$ Koho 1988;JP 63101365 A 19880506.

${ }^{22}$ Canli NY, Yildiz A, Karanlik G, Bilgin-Eran B, Okutan M. Conductivity Mechanism of Ethyl-4-(7,7,8,8,9,9,10,10,10-nonafluorodecyloxy)Biphenyl-4'-Carboxylate. J. Nanoelectron. Optoelectron. 2016;11:36-40.

${ }^{23}$ Yildiz A, Canli N, Karanlik G, Ocak H, Okutan M, Bilgin-Eran B. The determination of the phase transition temperatures of a semifluorinated liquid crystalline biphenyl ester by impedance spectroscopy as an alternative method. Physica B. 2016;503:152-156.

${ }^{24}$ Karanlik G, Ocak H, Bilgin-Eran B. New pyridine based liquid crystalline esters with different terminal chains. J. Mol. Struct. 2019;1198:126930-126938.

${ }^{25}$ Vardar D, Akdaş-Kiliç H, Ocak H, Jeannin O, Camerel F, Bilgin-Eran B, Pyridine Based Chiral Smectogens: Effects of Polar End Groups on liquid crystal Properties. Liq Cryst. 2020; doi:10.1080/02678292.2020.1799445.

${ }^{26}$ Huo E, Yang D, Zhang Z, Lu Z, Sun H, Xin X, Huang Y, Liu Y, Jiang Q. Synthesis and characterization of 4-dodecyloxyphenyl and (4'-dodecyloxy-4-biphenyl)methylene-substituted bispyrrolylvinylthiophene-based polysquaraines having good solubility and very low bandgap for light absorption. J. Appl. Polym. Sci. 2012;128:1632-1639.

${ }^{27}$ Heppke G, Lotzsch D, Morrb M, Ernst L. New chiral side chains for ferro- and antiferroelectric liquid crystals derived from the preen-gland wax of the domestic goose. J. Mater. Chem 1997;7:1993-1999.

${ }^{28}$ Goodby JW. Twisted and frustrated states of matter. Proc. R. Soc. 2012;A 468. 